\title{
Ras-ERK signaling in behavior: old questions and new perspectives
}

\author{
Stefania Fasano ${ }^{1}$ and Riccardo Brambilla ${ }^{1,2 *}$ \\ 1 Institute of Experimental Neurology, Division of Neuroscience, San Raffaele Scientific Institute and University, Milano, Italy \\ 2 School of Biosciences, Cardiff University, Cardiff, UK
}

\section{Edited by:}

Gilberto Fisone, Karolinska Institutet, Sweden

Reviewed by:

Rosario Moratalla, Cajal Institute, Consejo Superior de Investigaciones

Cientificas, Spain

Michael R. Bruchas, Washington

University in St. Louis, USA

*Correspondence:

Riccardo Brambilla, San Raffaele Scientific Institute and Vita-Salute San Raffaele University, Via Olgettina 58, 20132 Milano, Italy.

e-mail: brambilla.riccardo@hsr.it
The role of Ras-ERK signaling in behavioral plasticity is well established. Inhibition studies using the blood-brain barrier permeable drug SL327 have conclusively demonstrated that this neuronal cell signaling cascade is a crucial component of the synaptic machinery implicated in the formation of various forms of long-term memory, from spatial learning to fear and operant conditioning. However, abnormal Ras-ERK signaling has also been linked to a number of neuropsychiatric conditions, including mental retardation syndromes ("RASopathies"), drug addiction, and L-DOPA induced dyskinesia (LID). The work recently done on these brain disorders has pointed to previously underappreciated roles of RasERK in specific subsets of neurons, like GABAergic interneurons of the hippocampus or the cortex, as well as in the medium spiny neurons of the striatum. Here we will highlight the open questions related to Ras-ERK signaling in these behavioral manifestations and propose crucial experiments for the future.

Keywords: Ras, ERK, neurofibromin, ERK1, drug addiction, L-DOPA induced dyskinesia, RASopathies, Ras-GRF1
Since the discovery, 15 years ago, of the role of Ras-ERK signaling in long-term potentiation first (English and Sweatt, 1996, 1997) and then in memory formation (Brambilla et al., 1997; Silva et al., 1997; Atkins et al., 1998), a considerable experimental evidence has been accumulated to support the idea that this signal transduction pathway is crucial for strengthening synaptic connections in a variety of behavioral processes. The scope of this work is not to provide an extensive review of the available data but rather to highlight the aspects which still need to be clarified in order to gain a comprehensive description of the molecular processes involving Ras-ERK in behavior. For a detailed analysis of the published evidence, we refer to a few excellent review articles appeared in the last 4-5 years (Davis and Laroche, 2006; Girault et al., 2007; Santini et al., 2008; Samuels et al., 2009).

\section{THE RAS-ERK PARADOX: BALANCING SYNAPTIC EXCITATION AND INHIBITION}

Early on, when the first two publications on genetically altered mice in the Ras-ERK pathway became simultaneously available, what we call here the Ras-ERK paradox appeared immediately clear: any manipulation of this pathway either causing an enhancement or a partial inhibition of cell signaling, would lead to learning and memory deficits (Brambilla et al., 1997; Silva et al., 1997). In fact, not only the ablation from the mouse of Ras-GRF1, a CNS specific neuronal activator of Ras proteins, results in memory deficits but also the gene disruption of the neurofibromatosis type $1(\mathrm{NF} 1)$ gene, the locus responsible for the expression of the Ras-specific negative regulator neurofibromin, a GTPase activating protein, causes significant learning impairments. Although these early studies did not provide any biochemical evidence supporting the expected contrasting cell signaling effect of the two mutations (Ras-GRF1 loss would attenuate Ras activity while neurofibromin should enhance it), these genetic results initially suggested that any bidirectional alteration from the "physiological" neuronal ERK activity would negatively impact on the brain's ability to correctly process information. There are many example in which biological responses follow an "inverted U" shape, for instance the effect on memory mediated by corticosteroids, but the Ras-ERK paradox is a unique case in which the modulation of an intracellular signal transduction pathway results in such a clear detrimental effect on cognitive functions. This observation was clearly underappreciated at that time, but in retrospect it was and it is still a formidable obstacle to the development of cognitive enhancers which, either directly or indirectly, would impact on ERK signaling. One of the reasons why this question was not promptly investigated is that immediately after the Ras-GRF1 and NF1 KO publications, SL327 became available. This small chemical inhibitor of MEK1/2, the kinases upstream of ERK1/2, has been extremely useful in dissecting the effect of ERK inhibition on behavior since it rapidly passes the brain-blood barrier. Later on, other similar drugs started to be used, e.g., PD184161, with similar effects. Predictably, ERK inhibition does block longterm experience- and drug-dependent behavioral plasticity (see the above articles for review). In addition, this pharmacological approach has also been instrumental in demonstrating a central role of the ERK pathway in psychiatric conditions less dependent on memory functions such as chronic stress and depression (see for instance Duman et al., 2007). However, this plethora of inhibition studies, generated in the last decade, has not helped us much in resolving the ERK paradox but has simply confirmed that this pathway is a necessary ("permissive") condition to cause longterm changes behavior. Once again, the use of genetically altered animals has instead significantly contributed to define a much more complex role of Ras-ERK in the brain. In 2002 the initial 
hypothesis that a general and "non-cell-specific" increase in ERK signaling may lead to memory impairments had to be dismissed on the basis of two publications. Firstly, the ideal system to test this hypothesis has been represented by the ERK1 mutant mice which show a general enhancement of ERK activity in the brain (Mazzucchelli et al., 2002). This is due to a de-repression of ERK2, the main MAP kinase. In normal conditions, ERK1 acts as a built-in partial agonist, keeping ERK2 activity tightly regulated (Vantaggiato et al., 2006; Indrigo et al., 2010). Despite this general effect of the biochemistry of ERK2, ERK1 mutant animals do not show any sign of cognitive deterioration as one would have predicted from the early studies on the NF1 KO mice. On the contrary, ERK1 deficient mice manifest specific memory enhancing effects, including striatumdependent operant conditioning long-term memory formation, increased fear memory consolidation and extinction, novel object recognition memory, and cocaine-dependent associative and nonassociative learning but not spatial memory changes (Mazzucchelli et al., 2002; Cestari et al., 2006; Ferguson et al., 2006; Tronson et al., 2008; Berardi et al., 2011). Interestingly, while two independently generated Ras-GRF1 KO lines seem to show behavioral responses which are the mirror image of those found in the ERK1 mutants, a mutant showing a mild over-expression of Ras-GRF1 manifest a similar memory enhancing effect in most tasks but not, quite surprisingly, in spatial memory ones (Fasano et al., 2009a; Berardi et al., 2011; D'Isa et al., 2011). Thus, the results obtained with the analysis of the ERK1 mutants indicate that a general enhancement of Ras-ERK signaling can promote learning and memory functions. However, this observation is still in apparent contrast to the phenotype observed in the NF1 mice in which, supposedly, a general ERK enhancement occurs as well. The explanation of this discrepancy was provided by two papers in 2002 and in 2008, both from the Silva's lab. These two papers radically changed our view on cell signaling mediated by Ras-ERK in the brain since they clearly demonstrated that the Ras-dependent effect of neurofibromin ablation is specifically linked to an increase in GABA inhibition which is the likely cause of the learning and memory deficits. Either using the global heterozygous NF1 mice or cellspecific promoters to drive CRE expression in conditional NF1 mutants, Silva and co-workers showed that the behavioral effect was compatible with an enhancement of Ras activity in GABAergic interneurons that in turn may affect synaptic plasticity in key areas of the brain implicated in learning, such as the hippocampus. Interestingly, the memory deficits could be partially rescued by either reducing (pharmacologically or genetically) Ras activity (especially of the K-Ras isoform) or by partially inhibiting GABA activity using receptor antagonists. These experimental observations are important since they provide a likely explanation why loss of neurofibromin in NF1 patients may lead to learning disabilities. Importantly, they may also provide a rationale to understand other genetic diseases characterized by gain of function mutations in the Ras-ERK pathway leading to mental retardation, the now called RASopathies or Ras-MAPK syndromes (Aoki et al., 2008; Tidyman and Rauen, 2009). The clinical relevance of these disorders is such that a cogent question arising from the work on NF1 mutant mice requires an urgent answer: why do these changes in the RasERK activity only occur in GABAergic cells and not in all neurons? Indeed the data provided by the Silva's 2008 paper indicate that a mutation restricted to glutamatergic pyramidal cells, using the CamKII promoter-CRE line has no impact on behavior. Consistently, our own data on a conditional K-Ras G12V knock-in mutant line also show that a pyramidal specific activation of this Ras isoform has no effect on learning and memory (Papale et al., 2010). On the contrary, the CRE line driving expression under the Synapsin I early promoter leads to significant learning impairments in both NF1 and K-Ras12V mutants. In order to explain these observations, some possibilities arise. Firstly, expression analysis provided by the Allen Brain Atlas (www.brain-map.org) of both NF1 and K-Ras genes seems to indicate that their transcriptome levels are higher from late embryonic development (E18.5) to early post-natal stages ( $\mathrm{P} 4)$, a phase in which the Synapsin I early promoter is already active while the CamKII promoter is not. In addition, since during the post-natal maturation of GABAergic cells, the expression of NF1 and of K-Ras is maximal while later during pyramidal cell development is down to lower levels the occurrence an increased inhibition would be favored. Indeed, an indirect evidence that K-Ras is acting early in development during a temporal window which coincides with GABAergic development comes from the transgenic mouse model in which the H-RasG12V mutation is expressed under the CamKII promoter. In that case a forced over-expression in pyramidal neurons of this gain of function mutant leads to memory and plasticity improvements (Kushner et al., 2005; Kaneko et al., 2010). Whether this positive effect on memory is specific to this Ras isoform still needs to be seen but it is unlikely. Indeed, to complicate the matter, a recently described targeted expression of the very same H-RasG12V via homologous recombination (gene knock-in) results in significant behavioral impairments which partially recapitulate the phenotypes observed in patients affected by the Costello Syndrome, one of the RASopathies (Viosca et al., 2009). Thus, all available data point to the importance of "when" and "where": if Ras-ERK signaling is predominantly active in the GABAergic compartment, then plasticity and memory impairments may occur while the opposite is true when ERK activity is mainly activated in pyramidal cells. Indeed the real scenario is probably even more complex than that if we take into consideration the global ERK1 KO model in which memory improves despite gene ablation is not cell-type selective and the manipulation alters a protein hardly regulated in development. In this specific case, a possible explanation is that ERK1 loss causes a differential effect between excitation and inhibition by favoring the former. If that is the real case still remain to be seen since appropriated experiments, either using conditional ERK1 mutants or viral-mediated cell-specific gene knockdowns of ERK1, have not yet been performed. However, one prediction which can already be made is that a specific enhancement of ERK2 activity in GABAergic cells via ERK1 ablation should recapitulate the NF1 KO phenotype by bypassing the requirement for an elevated Ras activity. Certainly, this prediction could be incorrect in the case that other Ras-dependent signaling pathways, e.g., the PI3 Kinase cascade, significantly contribute to the mental retardation phenotype observed in the NF1 mutants.

One last comment is necessary on the exact mechanism by which neurofibromin and K-Ras cause the enhancement of GABAergic activity. Currently this is unclear but the possible explanations, not mutually exclusive are: (i) enhanced GABA 
release; (ii) larger GABA synapses; (iii) more GABA synapses; (iv) enhanced GABA-mediated post synaptic signaling. In any case, it is clear that in cortical regions and in the hippocampus, future experiments targeting the Ras-ERK signaling in a cell-specific manner and at different stages of development will provide substantial new evidence and will tell us whether the development of general cognitive enhancers based on ERK manipulation will ever be a viable therapeutic option for memory impairments.

\section{NEURONAL CELL SIGNALING BEYOND THE RAS-ERK PARADOX: THE STRANGE CASE OF THE STRIATUM}

Most cortical and hippocampal regions function by a tight integration of excitatory and inhibitory signals. Alterations of this balance, as we have seen above, may result in either a memory gain or a memory loss. However, not all brain areas work in this way. For instance, the striatum, the input nucleus of the basal ganglia system, is essentially constituted (>95\%) by GABAergic projection neurons, the so called medium spiny neurons (MSN; Kreitzer and Malenka, 2008). Instead of generating an output activity toward the thalamus and the motor cortex by balancing excitation and inhibition within its structure, the dorsal portion of the striatum (the ventral one, the nucleus accumbens, may be slightly different) integrates two main neurotransmitter signals, the glutamatergic and the dopaminergic ones, and conveys their action on two distinct subclasses of MSN: the direct pathway neurons, mainly expressing dopamine D1 receptors, and the indirect pathway neurons, mainly expressing D2 receptors. In normal conditions, a balanced activation of both pathways leads to an efficient activation of the thalamus and of the cortex. However, in certain neuropsychiatric diseases, one of the two pathways tends to dominate: an enhancement of the direct pathway leads to motor activation while that of the indirect pathway results in motor inhibition.

Not surprisingly, in recent years, the role of the Ras-ERK pathway and downstream gene expression has extensively been investigated in the striatum, using both pharmacological (e.g., SL327) or genetic approaches. The scenario which has resulted from these studies is that in both the behavioral responses to drugs of abuse ("drug addiction") and in a pathological condition resembling L-DOPA induced dyskinesia (LID) in Parkinson's Disease, an aberrant hyperactivation of Ras-ERK appears to be a key pathogenetic factor (Murer and Moratalla, 2011). In general terms, the most widely studied drugs of abuse, psychostimulants (e.g., cocaine and amphetamine), opiates (e.g., morphine and heroine), and cannabinoids (delta-9-tetrahydrocannabinol, THC) can cause both short-term changes of motor activity or long-term behavioral changes, which can include an enhanced locomotor activity or increased reward responses, as measured in conditioned place preference (CPP) or in self-administration procedures. The fact that pharmacological inhibition of ERK blocks these responses is now well established but, for the same reasons of the memory studies discussed in the previous chapter, it provides little additional information beyond establishing a "permissive" role of this pathway in the process.

On the contrary, mouse models of specific genes in the pathway have significantly contributed to outline an interesting scenario.
For instance, the original report on ERK1 KO mice that demonstrated an "instructive" role of ERK signaling in memory consolidation, i.e., these mice showed better striatum-dependent memory, also showed that CPP responses to morphine can be significantly enhanced (Mazzucchelli et al., 2002). This effect is not drug-specific since the same can be seen with cocaine (Ferguson et al., 2006). Similar drug-dependent enhancing effects have subsequently been seen in other mutant mice, most notably a striatal-specific dominant negative form of the transcription factor CREB ("killer CREB") and an overexpressing line for Ras-GRF1 (Fasano et al., 2009a,b). The case of CREB is particularly intriguing since the same dominant negative mutant, not only causes memory impairment when expressed in a "hippocampus" specific mouse line but also leads to instrumental learning deficits and LTP/LTD loss in a striatal-specific line (Pittenger et al., 2002, 2006). Thus, the manipulation of CREB within a given brain area, the dorsal striatum, shows a stimulus-specific effect which is in contrast to what was observed for other mutations in the ERK pathway, most notably Ras-GRF1, in which the gene disruption causes both memory loss and a reduced response to drugs (Brambilla et al., 1997; Fasano et al., 2009a; D'Isa et al., 2011). In the case of CREB, a likely possibility is that a repeated administration of a drug of abuse may lead to a compensatory upregulation of endogenous CREB, as seen previously for hypomorphic mutations of the gene itself or after viral-mediated expression of either WT or other dominant negative mutants of CREB in the Nucleus Accumbens (see Carlezon et al., 2005). It is obvious that more sophisticated experiments will be required to fully understand the role of CREB in striatum specific behavioral plasticity, including its expression specifically in either the direct or in the indirect pathway MSN.

In that respect, the use of $\mathrm{BAC}$ transgenic mice expressing the green fluorescent protein (GFP) in the two MSN compartments has enormously facilitated the analysis of the activation profile of the Ras-ERK pathway. Drugs like cocaine specifically activate ERK1/2 in the direct pathway MSN (Girault et al., 2007; Bertran-Gonzalez et al., 2008). On the contrary, antipsychotics like haloperidol, uniquely induced ERK1/2 activity in the indirect pathway. Consistently with the molecular data, a specific activation of the direct pathway leads to motor activation whereas motor inhibition is seen when the indirect pathway is induced.

L-DOPA induced dyskinesia is a severe condition in which chronic administration (several years) of the gold standard treatment of Parkinson's Disease results in abnormal involuntary movements (AIM). This pathological condition can be modeled in rodents by causing an unilateral loss of the substantia nigra pars compacta $(\mathrm{SNc})$ neurons with the neurotoxin 6-hydroxy dopamine (6-OHDA) followed by repeated L-DOPA injections. LID takes several years to appear in patients while in rodents the effect is almost immediate, after one or few injections of the therapeutic drug. In recent years, we have started to understand the molecular mechanisms underlying LID in rodents and the key event occurring in the dorsal portion of the striatum, the target region of the dopaminergic SNc cells, is the supersensitization of D1 receptor signaling through the upregulation of $G_{\text {olf }}$ (Girault et al., 2007). In other words, LID is essentially characterized by an aberrant enhancement of the striatal direct 
pathway, a feature, as we have seen, shared with drug addiction (Cenci, 2007; Jenner, 2008; Murer and Moratalla, 2011). The intriguing similarities between LID and the responses to drugs of abuse are also evident for what ERK signaling is concerned. Indeed, this signaling pathway is massively upregulated in the striatum of dyskinetic animals. To our knowledge, the induction of phosphorylated ERK1/2 in D1 receptor expressing MSN is the strongest ever detected not only in brain but in the whole body, accounting to up 50-fold increase over basal levels (as a comparison, high dose of cocaine can lead to a 10 -fold increase). This enormous activation of ERK signaling is a combination of the engagement of the larger share of D1R expressing MSN and also, to a lesser extent, of an increase of the signal in already activated cells (Gerfen et al., 2002; Pavon et al., 2006; Westin et al., 2007). The specificity of the cell-type implicated in ERK activation was initially demonstrated pharmacologically but then it was confirmed using the BAC transgenic mice mentioned above as well as by causing gene ablation of the D1 receptors (Santini et al., 2008, 2009; Darmopil et al., 2009). As expected from the initial observations, pharmacological inhibition of ERK using SL327 or a partial blockade of Ras activity using statins results in a significant attenuation of LID onset (Santini et al., 2007; Schuster et al., 2008). More recently, also the Ras-GRF1 mutant mouse which was previously shown to be involved in memory and in drug addiction has been used to test the role of this molecule in LID. Indeed, a strong reduction in the AIM profile of Ras-GRF1 KO animals was observed together with a very significant attenuation of ERK1/2 activity in the dorsal striatum of these animals (Fasano et al., 2010). Interestingly, that work also showed that a combined treatment with suboptimal doses of SL327 $(10 \mathrm{mg} / \mathrm{kg})$, a condition which per se is ineffective in wild-type animals, causes an almost complete reduction of the dyskinetic symptoms.

All these data strongly support the notion that a specific targeting of neuronal components of the Ras-ERK pathway, like Ras-GRF1, may lead to effective treatments of both drug addiction and LID. In that respect, the scientific community still lacks pharmacological tools which go beyond SL327 and can target other molecular components besides MEK1/2. An interesting option is the development of cell permeable peptides that readily cross the brain-blood barrier and cause reversible disruptions of proteinprotein interactions (Patel et al., 2007; Heitz et al., 2009). For instance, one can imagine that a Ras-GRF1 specific cell permeable peptide would be a valuable tool to study the role of this molecule in diverse behavioral processes, from memory formation, reconsolidation, and extinction, as well as for the development of treatments for the above mentioned diseases. At the same time, it is imperative to further advance in the development of cell-specific genetic tools to target crucial components of the Ras-ERK pathway with a tight temporal and spatial control. One step in that direction is the availability of BAC transgenics expressing CRE

\section{REFERENCES}

Aoki, Y., Niihori, T., Narumi, Y., Kure, S., and Matsubara, Y. (2008). The RAS/MAPK syndromes: novel roles of the RAS pathway in human genetic disorders. Hum. Mutat. 29, 992-1006.

Atkins, C. M., Selcher, J. C., Petraitis, J. J., Trzaskos, J. M., and Sweatt, J. D. (1998). The MAPK cascade is

recombinase in a cell and brain area specific manner. For instance, the recent report in which DARPP-32, a signal integrator which in the striatum also controls ERK signaling, was either specifically targeted in the MSN of the direct or the indirect pathway lead to changes in the responses to cocaine/L-DOPA or to haloperidol, respectively (Bateup et al., 2010). Also, a very significant leap forward would be represented by the combination of conditionally targeted mouse mutants with cell-specific CRE delivery via viral vectors which would also allow us to achieve an excellent temporal control of gene expression. At present though, since some limitations still apply to validate viral vectors with bona fide cell-specific promoters, probably the best option would be to use BAC transgenic lines expressing CRE in combination with vectors conditionally expressing either dominant negative constructs, cell permeable peptides, or small interfering RNAs (shRNA), as recently suggested (Papale et al., 2009). These multiple approaches should be able to address the major remaining question linking drug addiction, LID and the Ras-ERK pathway in the striatum: why is only the direct pathway affected in these diseases? The specificity certainly lies on the hyperactivation of the D1 receptors in response to L-DOPA or to drugs like cocaine but this type of reasoning is rather circular and provides little explanation. So far, there is no evidence that any component of the Ras-ERK pathway is differentially expressed in the two striatal cell populations. Thus, it is difficult to judge whether the activation of Ras-ERK in D1R expressing cells is just a "minor" consequence of upstream events or plays a more "instructive" role. Certainly, gene ablation of D1R completely block ERK activation and downstream events in the striatum and affects not only LID but also learning and LTP in the hippocampus, as well as cocaine self-administration (Caine et al., 2007; Darmopil et al., 2009; Ortiz et al., 2010). An interesting experiment in the direction to solve this problem would be to force over-expression of one of Ras-ERK elements, e.g., RasGRF1 (which is normally in both MSN types) in the indirect pathway, which is silent in LID and in response to cocaine, and see whether that is sufficient to readjust the system. In addition, it would be important to knockdown Ras-GRF1 individually in each compartment and to express constitutively active mutations in the pathway (e.g., Ras G12V) and to verify the effect at the behavioral level. Both loss and gain of function experiments will be crucial but certainly demanding from the technological point of view. However, in our opinion, they will represent a necessary new level of investigation to tackle the complexity of Ras-ERK signaling in behavior which will keep us busy for at least additional 15 years.

\section{ACKNOWLEDGMENTS}

This work was supported by the Michael J. Fox Foundation for Parkinson's Research and the Parkinson's UK as well as by the Italian Ministry of Health, the Fondazione CARIPLO and the Compagnia di San Paolo (to Riccardo Brambilla).

required for mammalian associative learning. Nat. Neurosci. 1, 602-609.

Bateup, H. S., Santini, E., Shen, W. Birnbaum, S., Valjent, E., Surmeier, D. J., Fisone, G., Nestler, E. J., and Greengard, P. (2010). Distinct subclasses of medium spiny neurons differentially regulate striatal motor behaviors. Proc. Natl. Acad. Sci. U.S.A. 107, 14845-14850. 
Berardi, N., Silingardi, D., Angelucci, A., De Pasquale, R., Borsotti, M., Squitieri, G., Putignano, E., Brambilla, R., and Pizzorusso, T. (2011). ERK pathway activation bidirectionally affects visual recognition memory and synaptic plasticity in the perirhinal cortex. Front. Behav. Neurosci. (in press).

Bertran-Gonzalez, J., Bosch, C., Maroteaux, M., Matamales, M. Herve, D., Valjent, E., and Girault, J. A. (2008). Opposing patterns of signaling activation in dopamine $\mathrm{D} 1$ and D2 receptor-expressing striatal neurons in response to cocaine and haloperidol. J. Neurosci. 28, 5671-5685.

Brambilla, R., Gnesutta, N., Minichiello, L., White, G., Roylance, A. J., Herron, C. E., Ramsey, M., Wolfer, D. P., Cestari, V., Rossi-Arnaud, C., Grant, S. G., Chapman, P. F., Lipp, H. P., Sturani, E., and Klein, R. (1997). A role for the Ras signalling pathway in synaptic transmission and longterm memory. Nature 390, 281-286.

Caine, S. B., Thomsen, M., Gabriel, K. I., Berkowitz, J. S., Gold, L. H., Koob, G. F., Tonegawa, S., Zhang, J., and $\mathrm{Xu}, \mathrm{M}$. (2007). Lack of self-administration of cocaine in dopamine D1 receptor knock-out mice. J. Neurosci. 27, 13140-13150.

Carlezon, W. A. Jr., Duman, R. S., and Nestler, E. J. (2005). The many faces of CREB. Trends Neurosci. 28, 436-445.

Cenci, M. A. (2007). Dopamine dysregulation of movement control in L-DOPA-induced dyskinesia. Trends Neurosci. 30, 236-243.

Cestari, V., Costanzi, M., Castellano, C., and Rossi-Arnaud, C. (2006). A role for ERK2 in reconsolidation of fear memories in mice. Neurobiol. Learn. Mem. 86, 133-143.

Darmopil, S., Martin, A. B., De Diego, I. R., Ares, S., and Moratalla, R. (2009). Genetic inactivation of dopamine D1 but not D2 receptors inhibits LDOPA-induced dyskinesia and histone activation. Biol. Psychiatry 66, 603-613.

Davis, S., and Laroche, S. (2006). Mitogen-activated protein kinase/extracellular regulated kinase signalling and memory stabilization: a review. Genes Brain Behav. 5(Suppl. 2), 61-72.

D'Isa, R., Clapcote, S. J., Voikar, V., Wolfer, D. P., Giese, K. P., Brambilla, R., and Fasano, S. (2011). Mice lacking Ras-GRF1 show contextual fear conditioning but not spatial memory impairments: convergent evidence from two independently generated mouse mutant lines. Front. Behav. Neurosci. 5:78. doi: $10.3389 /$ fnbeh.2011.00078
Duman, C. H., Schlesinger, L., Kodama, M., Russell, D. S., and Duman, R. S. (2007). A role for MAP kinase signaling in behavioral models of depression and antidepressant treatment Biol. Psychiatry 61, 661-670.

English, J. D., and Sweatt, J. D. (1996). Activation of p42 mitogen-activated protein kinase in hippocampal long term potentiation. J. Biol. Chem. 271, 24329-24332.

English, J. D., and Sweatt, J. D. (1997). A requirement for the mitogen-activated protein kinase cascade in hippocampal long term potentiation. J. Biol. Chem. 272, 19103-19106.

Fasano, S., Bezard, E., D'Antoni, A., Indrigo, M., Francardo, V., Qin, L., Dovero, S., Cerovic, M., Cenci, M. A., and Brambilla, R. (2010). Inhibition of Ras-GRF1 in the striatum reverts motor symptoms associated to L-DOPA induced Dyskinesia. Proc. Natl. Acad. Sci. U.S.A. 107, 21824-21829.

Fasano, S., D'Antoni, A., Orban, P. C., Valjent, E., Putignano, E., Vara, H., Pizzorusso, T., Giustetto, M., Yoon, B., Soloway, P., Maldonado, R., Caboche, J., and Brambilla, R. (2009a). Ras-guanine nucleotidereleasing factor 1 (Ras-GRF1) controls activation of extracellular signal-regulated kinase (ERK) signaling in the striatum and long-term behavioral responses to cocaine. Biol. Psychiatry 66, 758-768.

Fasano, S., Pittenger, C., and Brambilla, R. (2009b). Inhibition of CREB activity in the dorsal portion of the striatum potentiates behavioral responses to drugs of abuse. Front. Behav. Neurosci. 3:29. doi:10.3389/neuro.08.029.2009

Ferguson, S. M., Fasano, S., Yang, P., Brambilla, R., and Robinson, T. E. (2006). Knockout of ERK1 enhances cocaine-evoked immediate early gene expression and behavioral plasticity. Neuropsychopharmacology 31, 2660-2668.

Gerfen, C. R., Miyachi, S., Paletzki, R., and Brown, P. (2002). D1 dopamine receptor supersensitivity in the dopamine-depleted striatum Results from a switch in the regulation of ERK1/2/MAP kinase. $J$. Neurosci. 22, 5042-5054.

Girault, J. A., Valjent, E., Caboche, J., and Herve, D. (2007). ERK2: a logical AND gate critical for drug-induced plasticity? Curr. Opin. Pharmacol. 7 , $77-85$.

Heitz, F., Morris, M. C., and Divita, G. (2009). Twenty years of cellpenetrating peptides: from molecular mechanisms to therapeutics. $\mathrm{Br}$. J. Pharmacol. 157, 195-206.
Indrigo, M., Papale, A., Orellana, D., and Brambilla, R. (2010). Lentiviral vectors to study the differential function of ERK1 and ERK2 MAP kinases. Methods Mol. Biol. 661, 205-220.

Jenner, P. (2008). Molecular mechanisms of L-DOPA-induced dyskinesia. Nat. Rev. Neurosci. 9, 665-677.

Kaneko, M., Cheetham, C. E., Lee, Y S., Silva, A. J., Stryker, M. P., and Fox, K. (2010). Constitutively active $\mathrm{H}$-ras accelerates multiple forms of plasticity in developing visual cortex. Proc. Natl. Acad. Sci. U.S.A. 107 19026-19031.

Kreitzer, A. C., and Malenka, R. C. (2008). Striatal plasticity and basal ganglia circuit function. Neuron 60 , 543-554.

Kushner, S. A., Elgersma, Y., Murphy, G. G., Jaarsma, D., van Woerden, G. M., Hojjati, M. R., Cui, Y., LeBoutillier, J. C., Marrone, D. F., Choi, E. S., De Zeeuw, C. I., Petit, T. L., Pozzo-Miller, L., and Silva, A. J. (2005). Modulation of presynaptic plasticity and learning by the $\mathrm{H}$-ras/extracellular signal-regulated kinase/synapsin I signaling pathway. J. Neurosci. 25, 9721-9734.

Mazzucchelli, C., Vantaggiato, C. Ciamei, A., Fasano, S., Porrazzo, A., Orban, P. C., Pakhotin, P., Krezel, W., Wezl, H., Wolfer, D. P., Pagès, G., Valverde, O., Marowsky, A., Porrazzo, A., Orban, P. C., Maldonado, R., Ehrengruber, M. U., Cestari, V., Lipp, H. P., Chapman, P. F., Pouysségur, J., and Brambilla, R. (2002). Knockout of ERK1 MAP kinase enhances synaptic plasticity in the striatum and facilitates striatal-mediated learning and memory. Neuron 34, 807-820.

Murer, M. G., and Moratalla, R. (2011). Striatal signaling in LDOPA-induced dyskinesia: common mechanisms with drug abuse and long term memory involving D1 dopamine receptor stimulation. Front. Neuroanat. 5:51. doi:10.3389/fnana.2011.00051

Ortiz, O., Delgado-Garcia, J. M., Espadas, I., Bahi, A., Trullas, R., Dreyer, J. L., Gruart, A., and Moratalla, R. (2010). Associative learning and CA3-CA1 synaptic plasticity are impaired in DIR null, Drd1a-/- mice and in hippocampal siRNA silenced Drdla mice. $J$. Neurosci. 30, 12288-12300.

Papale, A., Cerovic, M., and Brambilla, R. (2009). Viral vector approaches to modify gene expression in the brain. J. Neurosci. Methods 185, 1-14.

Papale, A., D'Isa, R., Solari, N., Formentini, I., Cerovic, M., Hardingham, N., Gangarossa, G., Fisone, G., and Brambilla, R. (2010). "A mouse model of cognitive impairments associated to the Ras-MAPK syndromes," in FENS Forum Abstract 057.66, Amsterdam.

Patel, L. N., Zaro, J. L., and Shen, W. C. (2007). Cell penetrating peptides: intracellular pathways and pharmaceutical perspectives. Pharm. Res. 24 1977-1992.

Pavon, N., Martin, A. B., Mendialdua, A., and Moratalla, R. (2006). ERK phosphorylation and FosB expression are associated with L-DOPAinduced dyskinesia in hemiparkinsonian mice. Biol. Psychiatry 59, 64-74.

Pittenger, C., Fasano, S., MazzocchiJones, D., Dunnett, S. B., Kandel, E. R., and Brambilla, R. (2006). Impaired bidirectional synaptic plasticity and procedural memory formation in striatum-specific cAMP response element-binding protein-deficient mice. J. Neurosci. 26, 2808-2813.

Pittenger, C., Huang, Y. Y., Paletzki, R. F., Bourtchouladze, R., Scanlin, H., Vronskaya, S., and Kandel, E. R. (2002). Reversible inhibition of CREB/ATF transcription factors in region CAl of the dorsal hippocampus disrupts hippocampusdependent spatial memory. Neuron $34,447-462$.

Samuels, I. S., Saitta, S. C., and Landreth, G. E. (2009). MAP'ing CNS development and cognition: an ERKsome process. Neuron 61, 160-167.

Santini, E., Alcacer, C., Cacciatore, S., Heiman, M., Herve, D., Greengard P., Girault, J. A., Valjent, E., and Fisone, G. (2009). L-DOPA activates ERK signaling and phosphorylates histone $\mathrm{H} 3$ in the striatonigral medium spiny neurons of hemiparkinsonian mice. J. Neurochem. 108, 621-633.

Santini, E., Valjent, E., and Fisone, G. (2008). Parkinson's disease: levodopa-induced dyskinesia and signal transduction. FEBS J. 275 , 1392-1399.

Santini, E., Valjent, E., Usiello, A., Carta, M., Borgkvist, A., Girault, J. A., Herve, D., Greengard, P., and Fisone, G. (2007). Critical involvement of cAMP/DARPP-32 and extracellular signal-regulated protein kinase signaling in L-DOPAinduced dyskinesia. J. Neurosci. 27, 6995-7005.

Schuster, S., Nadjar, A., Guo, J. T., Li, Q., Ittrich, C., Hengerer, B., and Bezard, E. (2008). The 3-hydroxy3-methylglutaryl-CoA reductase inhibitor lovastatin reduces severity of L-DOPA-induced abnormal involuntary movements in experimental Parkinson's disease. $J$. Neurosci. 28, 4311-4316. 
Silva, A. J., Frankland, P. W., Marowitz, Z., Friedman, E., Lazlo, G., Cioffi, D., Jacks, T., and Bourtchuladze, R. (1997). A mouse model for the learning and memory deficits associated with neurofibromatosis type I. Nat. Genet. 15, 281-284.

Tidyman, W. E., and Rauen, K. A. (2009). The RASopathies: developmental syndromes of Ras/MAPK pathway dysregulation. Curr. Opin. Genet. Dev. 19, 230-236.

Tronson, N. C., Schrick, C., Fischer, A., Sananbenesi, F., Pages, G., Pouyssegur, J., and Radulovic, J. (2008). Regulatory mechanisms of fear extinction and depression-like behavior. Neuropsychopharmacology 33, 1570-1583.

Vantaggiato, C., Formentini, I., Bondanza, A., Bonini, C., Naldini, L., and Brambilla, R. (2006). ERK1 and ERK2 mitogen-activated protein kinases affect Ras-dependent cell signaling differentially. J. Biol. 5, 14.

Viosca, J., Schuhmacher, A. J., Guerra, C., and Barco, A. (2009). Germline expression of $\mathrm{H}-\mathrm{Ras}(\mathrm{G} 12 \mathrm{~V})$ causes neurological deficits associated to Costello syndrome. Genes Brain Behav. 8, 60-71.
Westin, J. E., Vercammen, L., Strome, E. M., Konradi, C., and Cenci, M. A. (2007). Spatiotemporal pattern of striatal ERK1/2 phosphorylation in a rat model of L-DOPA-induced dyskinesia and the role of dopamine D1 receptors. Biol. Psychiatry 62, 800-810.

Conflict of Interest Statement: The authors declare that the research was conducted in the absence of any commercial or financial relationships that could be construed as a potential conflict of interest.
Received: 02 August 2011; paper pending published: 19 August 2011; accepted: 03 November 2011; published online: 24 November 2011.

Citation: Fasano $S$ and Brambilla $R$ (2011) Ras-ERK signaling in behavior: old questions and new perspectives. Front. Behav. Neurosci. 5:79. doi: 10.3389/fnbeh.2011.00079

Copyright (C) 2011 Fasano and Brambilla. This is an open-access article subject to a non-exclusive license between the authors and Frontiers Media SA, which permits use, distribution and reproduction in other forums, provided the original authors and source are credited and other Frontiers conditions are complied with. 